\title{
入侵植物凤眼蓝繁育系统在中国境内的地理变异
}

\author{
任明迅 ${ }^{1,2}$ 张全国 ${ }^{1}$ 张大勇 $^{1 *}$ \\ (1 北京师范大学生态学研究所, 生物多样性与生态工程教育部重点实验室 北京 100875) \\ (2 中国科学院植物研究所系统与进化植物学重点实验室 北京 100093)
}

\begin{abstract}
摘 要 对中国境内的凤眼蓝 (Eichhornia crassipes) 进行了大规模地理范围的调查, 分析了 24 个地点 40 个种群的克 隆繁殖与有性繁殖的相对水平、三型花柱的花型变化以及花部综合特征的变异。40 个种群都以克隆繁殖为主要更 新方式。其中 36 个种群只有三型花柱中的 M 花型; 南宁、重庆的种群中出现了频率极低的 L 花型; 南宁、重庆、福 州和海口种群内, 出现了具有促进花内自交潜能的半同长花柱花型(柱头与花药相靠拢的 $\mathrm{M}$ 花型变异体或 $\mathrm{L}$ 花型 变异体)。与其它地方种群相比, 西南和海南岛的种群表现出较多样化的繁育系统。M 和 L 花型个体的结实个体 率都约为 $4 \%$ 没有显著差异 种子产量在两花型个体之间也没有差异。由于种群内花型的残缺, 作为促进花型之 间异交传粉适应机制的三型花柱繁育系统已经解体, 部分种群中出现了可以促进花内自交的半同长花柱变异体。 克隆繁殖是凤眼蓝适应入侵生境的一种形式, 花内自交是凤眼蓝繁育系统在入侵过程中可能的进化方向, 这些繁 育系统上的变化对该植物的入侵机制和途径产生了深远影响。
\end{abstract}

关键词 凤眼蓝 繁育系统 生物入侵 进化

\section{GEOGRAPHICAL VARIATION IN THE BREEDING SYSTEMS OF AN INVASIVE PLANT , EICHHORNIA CRASSIPES, WITHIN CHINA}

\author{
REN Ming-Xun ${ }^{1}{ }^{2}$ ZHANG Quan-Guo ${ }^{1}$ and ZHANG Da-Yong ${ }^{1 *}$ \\ (1 Ministry of Education Key Laboratory for Biodiversity Science and Ecological Engineering \& Institute of Ecology, \\ Beijing Normal University , Beijing 100875 , China) \\ (2 Laboratory of Systematic and Evolutionary Botany, Institute of Botany , Chinese Academy of Sciences , Beijing 100093 , China)
}

\begin{abstract}
Knowledge about the breeding systems of invasive weeds is crucial for invasion management. Eichhornia crassipes, a noxious clonal plant, has a complex breeding system that includes clonal reproduction and a special sexual reproduction mode, tristyly, and a complex mating system that consists of three floral morphs that differ in the length of styly and stamens. In this paper, we compared the breeding systems of 40 Eichhornia crassipes populations in 24 localities throughout China by investigating the relative level of sexual and clonal reproduction, number of floral morphs and the variances in floral syndrome (especially the position of sexual organs) for each population. A Mann-Whitney test was used to detect whether the variation in the position of the sexual organs were significant among each other and the typical floral morphs. The seed-set ratio of each floral morph was investigated extensively in a Chongqing population that had a high number of floral morphs.

All the populations were regenerated primarily by clonal propagation as a result of vigorous clonal growth. Sexual reproduction was presumably constrained because only one or two floral morphs existed in the populations. Sexual recruitment was further restricted by lack of suitable ecological conditions for seed germination and seedling establishment. Exposure to the sunlight and water temperature above $30{ }^{\circ} \mathrm{C}$ is generally necessary for seed germination, a condition not met in most populations.

Most of the populations surveyed were monomorphic for the $\mathrm{M}$ floral morph, and only the populations in Chongqing (West China on the Yangtze River) and Nanning (Southwest China near the tropical zone) contained the $\mathrm{L}$ morph with the M morph still predominating the populations. No significant differences in the fecundity of the M and L morphs were found and only $4 \%$ of the individuals produced seeds for these two floral morphs. The number of seeds per capsule varied greatly in both morphs, from 13 to 296 in the L morph and from 20 to 230 in the M morph , which suggested that fecundity was greatly restricted by ecological conditions , such as pollination and weather conditions, rather than developmental deficiency.

The variations in the position of the sexual organs in the $\mathrm{M}$ floral morph were not significantly different among each other and the typical flowers. But, populations in Chongqing, Nanning, Fuzhou and Haikou were
\end{abstract}


found to have modified M morphs or L morphs with anthers and stigma adjacent to each other (namely semi-homostylous flowers). Semi-homostyly is thought to be controlled by modifier genes and is selected for since it can increase fecundity through autogamy in invaded regions that have unreliable and inefficient pollinators. Based on the relative levels of sexual reproduction and number of floral morphs in a population, our results showed that the breeding systems of populations in the southwestern region and on Hainan Island were much more diverse than in other regions.

The evolutionary trend of the breeding systems that occurred in some $E$. crassipes populations is consistent with the Baker's law , which states that autogamy would be favored during an invasion event. These evolutionary changes of the breeding systems also have significant effects on the invasion process of this species.

Key words Eichhornia crassipes, Breeding system, Biological invasion, Evolution

植物入侵对自然生态系统和人类经济造成了严 重的破坏和不良影响，已经成为生态学、环境科学的 研究焦点。克隆植物 (Clonal plant) 是最为常见的入 侵植物类群, 这是因为克隆植物往往生长快速、表型 可塑性大、具有很强的生境适应能力, 且不依赖周期 漫长的有性繁殖更新后代, 很容易在新的分布区存 活和扩散 (Mack et al. 2000 ;Sax \& Brown , 2000 ;李 博等, 2001 徐承远等,2001 李振宇等 ,2002)。

植物的入侵过程也是该植物入侵个体适应新生 境的过程, 往往伴随着生长和繁殖方面快速的适应 性变化, 因此, 植物入侵现象也为研究植物繁育系统 的进化提供了绝佳机会 (Baker ,1974;Lee ,2002)。繁 育系统是指控制种群内有性繁殖或无性繁殖、异体 授精或自体授精相对频率的各种生理、形态机制 (Heywood,1979) , 是植物遗传机制在与外部环境条 件相互作用过程中的表现形式, 是决定植物适应能 力的关键, 也在很大程度上决定了植物进化路线和 表型变异模式。对于一些有着复杂繁育系统, 尤其 是复杂的有性繁殖方式(如不同个体有着不同有性 繁殖特征的雌雄异株 (Dioecy)、异长花柱 (Heterostyly)等)的入侵克隆植物来说，研究这些复杂系统在 入侵过程中的适应性变化具有重要的理论意义和实 践指导价值。根据 Silvertown 和 Charlesworth(2001) 的分析, 描述一种植物的繁育系统至少包括 3 方面 的内容:1)种群是无性繁殖的还是有性繁殖的?2) 如果是有性繁殖的, 是自交为主, 还是异交为主? 花 的性别特征 (性系统) 是什么?3) 影响植物自交和异 交的花部性状有哪些?由于繁育系统不仅在入侵过 程中发生了适应性变化, 也反过来对植物入侵机制、 途径等有着深刻的影响, 因此, 研究入侵植物的繁育 系统变化不但可以了解植物繁育系统的进化模式, 也是生物入侵研究和管理实践中的核心问题之一。

凤眼蓝 (Eichhornia crassipes) , 又名 水葫芦”, 属 雨久花科漂浮生长的多年生草本植物。凤眼蓝原产 南美亚马逊流域 现广布于世界热带、亚热带和温带
的淡水水域，被认为是最具入侵能力的恶性水生杂 草(Barrett ,1989；1992)。在中国, 曾经的人为引种 和广泛栽培造成凤眼蓝入侵了南方的大面积水域， 已经引发一系列严重的生态问题和经济损失 (Ding et al. 2000)。凤眼蓝的繁育系统十分复杂, 既有着 旺盛的、依靠匍匐茎实现的克隆繁殖, 也有着复杂的 有性繁殖方式一三型花柱 (Tristyly)。三型花柱是 由种群内 3 种不同花型个体组成的。3 种花型都是 雌雄同花 (Hermaphrodite) 柱头和花药位置在 3 种花 型中各不相同, 存在着交互对应的关系。同一花内 的性器官位置各异, 接触昆虫的不同部位, 从而避免 了花内花粉接受和散发的相互干扰; 不同花型之间 交互对应的异性器官接触到传粉昆虫身体的同一部 位而实现了准确传粉 (图 1)。三型花柱的结构十分 复杂而特别，几乎是植物适应昆虫传粉所能达到的 形态学复杂性的上限 (Barrett, 2002)。从达尔文的 开创性工作 (Darwin, 1877) 以来,三型花柱一直是遗 传学、植物繁殖生态学和进化生物学的一个引人注 目的研究模式(Barrett, 1990;1993)。

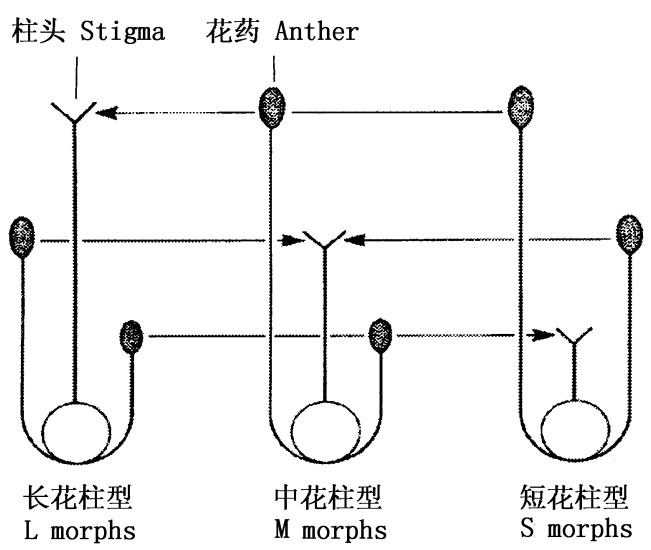

图 1 三型花柱

Fig.1 Tristylous breeding system

图中箭头示亲和的传粉方向 The arrows indicate the compatible pollinations

由于入侵的奠基者效应 (Founder' s effect), 凤眼 
蓝的三型花柱在其入侵地种群往往只有其中的 1 个 或 2 个花型。虽然对这种花型变化有过研究 (Barrett ,1977;1985)，但是，还没有从繁育系统角度来综 合分析凤眼蓝入侵过程中的有性繁殖与克隆繁殖相 对水平、花型数量与比率以及各花型内花部综合特 征 (Floral syndrome)的变化。尤其是在广衰的中国境 内 ,凤眼蓝种群的三型花柱、花部综合特征以及自然 环境下的有性繁殖水平及其对该植物成功入侵的贡 献等还没有开展过研究。我们大范围地调查了中国 分布区的凤眼蓝, 对上述一些问题进行了初步分析， 并通过繁育系统在入侵过程 (不同入侵种群)中的变 化探讨了凤眼蓝繁育系统的进化模式及这些进化改 变在该植物成功入侵中的地位。

\section{1 材料和方法}

在 2001、2002、2003 三年的夏季踏查了中国境内 的凤眼蓝分布区。由于凤眼蓝主要分布在我国的南 方地区, 尤其是长江流域及以南, 采样区域主要以长 江流域及其以南地区为主, 并对凤眼蓝入侵情况极 其严重的地点，如上海、武汉、福州、南宁、昆明和海 口等地, 进行了多种群的密集采样分析。其它采样 地点基本按照水系特点 ( 凤眼蓝是容易随波逐流的 水生飘浮植物) 和省份划分 (凤眼蓝曾经在不同省份 受到推广栽培)的标准选择。周泽江等(1984)指出, 北京和东北的盘锦和锦州一带也发现有凤眼蓝的野 外种群，所以我们也调查了这些地点。因此, 本研究 共对我国 24 个地点的 40 个凤眼蓝种群进行了调 查、采样。调查地点和种群分布见图 2 。

在每个种群内, 对整个种群范围内的不少于 100 朵花进行花内性器官位置的普查和测定, 区分 花型 M、L 或花部综合特征的任何显著变异, 并估测 各花型的比例。如果整个种群是单一的花型, 在整 个种群内随机选取 5 个开花个体, 每个个体花序上 选取上、中、下 3 花分别进行花内性器官位置 (从子 房基部计量长雄芯群的长度、花柱长度和短雄芯群 长度)的准确测量并记录。如果种群是多花型 (包括 显著变异了的 $M$ 花型或 $L$ 花型) 的, 对每个花型都 进行同上的测量记录。花型内的性器官位置数据进 行 Mann-Whitney 检验, 以确定这种变异之间以及与 典型的花型之间是否有着显著的差异。

传粉生物学观察:在调查花型的同时对传粉昆 虫的类型、访问方式与频率进行观察记录。凤眼蓝 单花花期不足 $1 \mathrm{~d}$ 花在早上 9:00 左右完全打开 (气 温降低、降水将延迟开花), 大约能持续开放到下午
4:00。传粉生物学的观察集中在早上 10:00 11:00 和下午 $2: 00 \sim 3: 00$ 进行。

有性繁殖水平调查: 大范围调查凤眼蓝植株果 序的结实率和蒴果结实情况，对于不同花型进行分 别调查。在每个种群的临岸处注意观察、搜寻有无 凤眼蓝的实生苗。

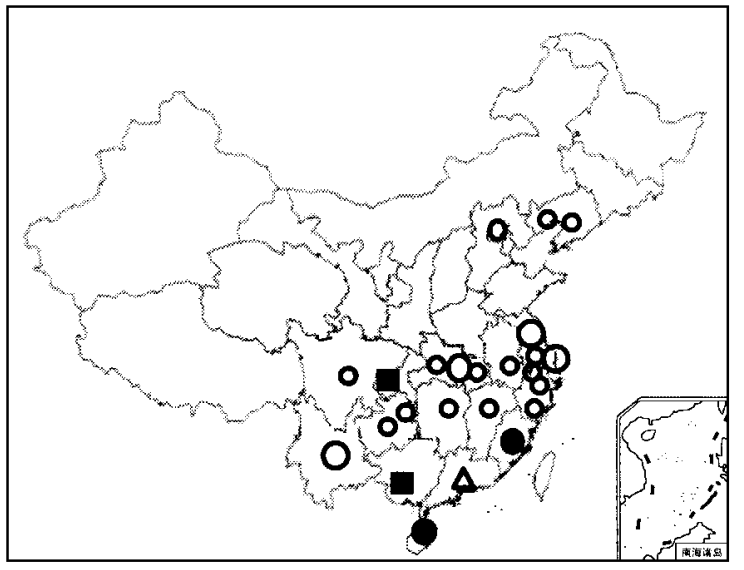

- 1 个调查种群 (花型M)

One population surveyed (M morphs)

O 3 个调查种群 (花型M)

Three populations surveyed (M morphs)

- 3 个调查种群 (花型 $\mathrm{M}+\mathrm{SH}$ )

Three populations surveyed (M+SH morphs)

- 3 个调查种群 (花型 $\mathrm{M}+\mathrm{L}+\mathrm{SH}$ )

Three populations surveyed (M+L+SH morphs)

$\Delta$ 调查期间无开花个体

No flowering individuals during the survey

图 2 调查的种群及其花型情况

Fig.2 The distribution and floral morphs of populations surveyed

M、L 和 SH 三种花型详见正文 The three kinds of floral morphs , M , $\mathrm{L}$ and $\mathrm{SH}$ are explained in the text

\section{2 实验结果}

\section{1 克隆繁殖与有性繁殖}

在调查的种群中, 广州种群在调查期间没有开 花个体 种群主要是进行克隆繁殖。在其它地方的 开花种群中, 大部分个体也都发现有数量较多的克 隆分株。通常一个母体植株能克隆发生出 10 个以 上的分株和次级分株 (分株产生的分株)。因此，从 克隆分株数量来看，所有种群中的克隆繁殖都很旺 盛。

对每个开花种群进行的广泛采集调查和子房解 剖发现 绝大多数花并没有结实。成熟果序( 凤眼蓝 花序在成熟后都会自然弯曲, 将果序带入水面以下 发育)的子房内胚珠大多完全败育。对花的解剖分 析发现, 凤眼蓝的雌雄生殖器官都发育良好，子房、 花药结构正常,胚珠败育的主要原因可能是不良的 生态条件 ,尤其是传粉条件所致。对重庆种群进行 
详尽的研究发现, M 和 L 花型个体的结实率都只有 $4 \%$ 左右, 每一果序仅有 1 个或 2 个蒴果结实, 每个 蒴果里的种子数量在 $13 \sim 296$ 粒之间, 种子产量变 化极大(表 1)。

在绝大多数种群内都没有发现实生苗。仅在海 南岛的海口附近 1 个种群 (海口共调查了 3 个种群) 的岸边 发现了种子萌发而来的实生苗。

\section{2 凤眼蓝的花部综合特征}

凤眼蓝的花为两性花, 花被基部结合成短管，外 面近基部被腺毛。花被裂片 6 片，上面 1 片较大，中 央具深蓝色块斑, 玟王中有着一颇似丹凤眼的鲜黄色 眼点, 是为蜜导 (Nectar guide)。雄荵 6 枚, 3 长 3 短, 着生在花被管上。花柱单一, 线性。花内的雌雄异 位 (Herkogamy) 程度较大(任明迅等,2004)。花丝和 花柱末端都略向上方蜷曲, 使得花药与柱头偏向蜜 导一面。花淡蓝紫色, 直径约 $3 \mathrm{~cm}$ 。每朵花通常在 清晨开放, 花裂片完全打开后数小时有花药开裂, 过 午后有部分自然散落。单花花期小于 $1 \mathrm{~d}$ 。

凤眼蓝花序为穗状花序, 通常有花 10 朵以上。 花序内花的开放顺序是自下向上，多朵花同时开放。 花序一般在 2 d 内开完。

\section{3 花型}

中国境内的凤眼蓝种群绝大多数都是只有 $\mathrm{M}$ 花型的单一花型种群, 仅在南宁和重庆两地的部分 种群内发现了少量的 L 型花(图 2), 与 Barrett(1989) 的预测相吻合。图 3 是各个种群 $\mathrm{M}$ 花型内的性器 官位置示意图(广州种群没有开花, 南宁种群在调查 期间只开 L 花, 所以这两个种群缺少花型数据)。

与三型花柱的典型花部结构 (图 1) 相比, 大多 数种群内的 $M$ 花型以及南宁种群的 $\mathrm{L}$ 花型, 其花内 性器官的位置有一定的变化: 柱头和雄芯群之间的 距离缩小。这种变异花型在该植物全球范围的入侵 种群中都有发现, 如哥斯达黎加 (Barrett, 1979)。 Barrett (1979) 把这种花药与柱头发生靠拢趋势变化 的变异花型都称为 半同长花柱” ( Semi-homostyly, $\mathrm{SH})$ 。

我们对 半同长花柱” 花型内性器官位置数据的 Mann-Whitney 检验发现, 这种性器官位置的变化并 不显著 $(p>0.3)$ 。这种微小变化难以确定与相互 比较, 也难以准确表明其引起的生态学和遗传学后 果。因此, 在这里, 我们只把花药或柱头位置变化很 大, 使得柱头和花药紧密靠拢、利于自交传粉的变异 花型称为 半同长花柱”。根据这种定义, 半同长花 柱只出现在福州、南宁、海口和重庆的种群内。

表 1 重庆种群 M 花型与 $\mathrm{L}$ 花型个体的结实情况

Table 1 The fecundity of $\mathrm{M}$ and $\mathrm{L}$ morph individuals in Chongqing population

\begin{tabular}{|c|c|c|c|c|c|c|c|}
\hline & \multirow{2}{*}{$\begin{array}{l}\text { 采集果序数 } \\
\text { Infructescences } \\
\text { investigated }\end{array}$} & \multirow{2}{*}{$\begin{array}{l}\text { 结实果序数 } \\
\text { Infructescences } \\
\text { producing seeds }\end{array}$} & \multirow{2}{*}{$\begin{array}{c}\text { 结实果序率 } \\
\text { Percentage of infructescences } \\
\text { producing seeds }(\%)\end{array}$} & \multicolumn{4}{|c|}{$\begin{array}{l}\text { 结实果序的种子产量 }(\text { 粒 }) \\
\text { No. of seeds produced per infructescence }\end{array}$} \\
\hline & & & & $\begin{array}{c}\text { 果序 } 1 \\
\text { Intructescence } 1\end{array}$ & $\begin{array}{c}\text { 果序 } 2 \\
\text { Intructescence } 2\end{array}$ & $\begin{array}{c}\text { 果序 } 3 \\
\text { Intructescence } 3\end{array}$ & $\begin{array}{c}\text { 果序 } 4 \\
\text { Intructescence } 4\end{array}$ \\
\hline M & 101 & 4 & 3.96 & 230 & 213 & 20 & 199 \\
\hline $\mathrm{L}$ & 50 & 2 & 4 & 13 & 296 & & \\
\hline
\end{tabular}

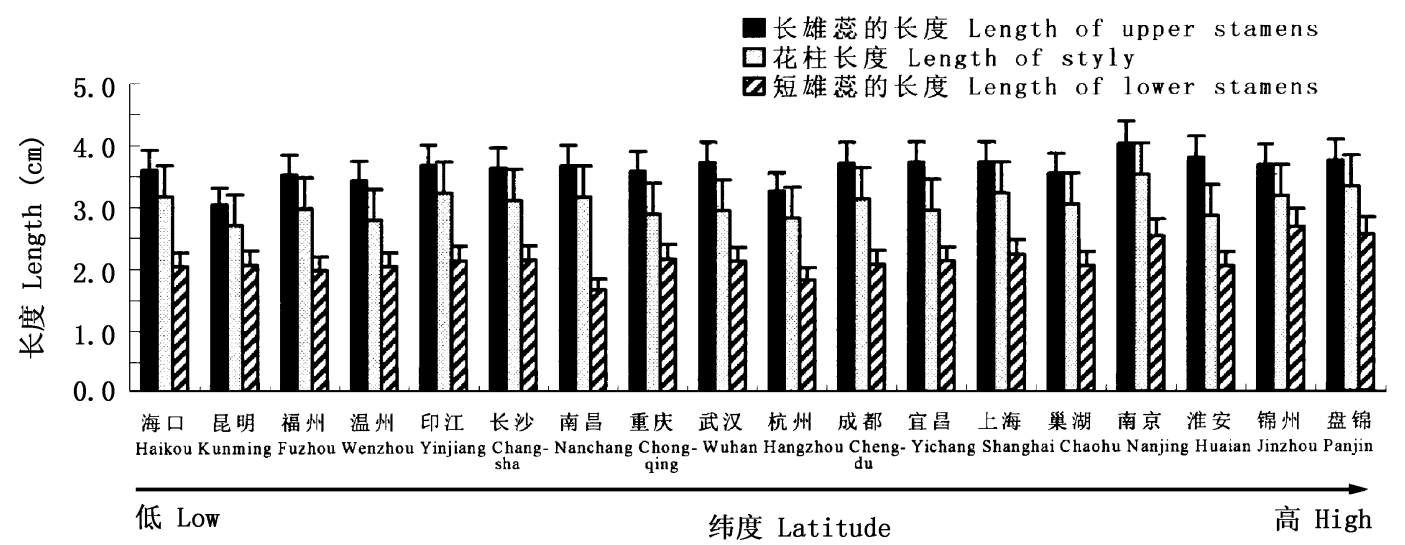

图 3 各地 $\mathrm{M}$ 花性器官位置的变异

Fig.3 The position of sexual organs in M flower of each population 


\section{3 讨 论}

\section{1 有性与克隆繁殖水平的相对水平}

凤眼蓝种群的主要更新方式是克隆繁殖, 这是 大多数水生植物的一个共同特点 (Grace, 1993 郭友 好等 ,1998)。凤眼蓝的原产地是位于南美亚马逊流 域的短暂性的浅水水体和泛滥洼地，凤眼蓝在这种 生境条件下进化出了漂浮水面和克隆繁殖的适应方 式，并通过极其快速、旺盛的克隆繁殖最大化克隆的 扩散速率, 而不是像陆生克隆植物是以克隆繁殖实 现空间利用的最优化 (Watson \& Cook ,1982)。正是 这种特性, 使得凤眼蓝在入侵地能以克隆繁殖为种 群的主要更新方式,并具有极强的入侵能力。

凤眼蓝在人工授粉后的种子产量可达 300 粒种 子/蒴果(Barrett ,1980a;1980b)。在重庆种群中也发 现, $\mathrm{M}$ 和 $\mathrm{L}$ 花型个体中部分蒴果的种子产量也接近 300 ,但也有蒴果只有 10 余粒或不到 200 粒的种子 (表 1)。这些偏低的结实量可能是由于生态条件 (如传粉)受到限制造成的。我们在多个地方种群进 行的传粉生物学观察发现, 大多数种群的访问昆虫 主要为小型的蜂类和蝇类, 往往只在一个雄芯群上 活动采集花粉, 不可能出现同时接触到花内的 3 个 性器官位置的现象, 难以实现三型花柱所适应的异 交传粉过程。这与 Barrett(1980a;1980b)以及唐佩华 等(1987)的研究结果相一致。

对 M 和 L 花型的花内雌雄异位的比较测量可 以发现, $\mathrm{M}$ 花具有较小的雌雄异位程度。由于较小 的雌雄异位能提高花内自交的可能性，凤眼蓝同时 也有极高的自交亲和性( Barrett ,1979；1993) ,所以， 自然种群中 M 花的自交更容易发生。Barrett (1977； 1989) 认为, 这是造成全球凤眼蓝种群都以 M 花型 为主 (有许多地方种群甚至只有 $M$ 花型) 的主要原 因。类似地“, 半同长花柱” 变异个体的雌雄异位程 度很小，在入侵地也将有着一定的选择优势。

由于 $\mathrm{M}$ 和 $\mathrm{L}$ 花型内性器官的对应排列, 有着这 两种花型的重庆种群和南宁种群很可能存在着花型 之间的异交传粉, 也许会出现较高的遗传变异。遗 传多样性的数据表明 ${ }^{1)}$, 有着 M、L 花型的种群的确 有着较其它种群更高的遗传变异程度。因此, 有性 繁殖可能在这些种群的入侵过程中起着一定的促进 作用，造成该植物在该地入侵态势的进一步恶化。
野外观察发现, 种子萌发和幼苗建成缺少合适 的生态条件也是限制凤眼蓝有性繁殖的一个主要原 因。凤眼蓝种子萌发的条件需要光照和 $30{ }^{\circ} \mathrm{C}$ 以上 的水温(Barrett, 1977;1989) ,这些条件在许多入侵地 都难以实现或难以维持足够长的时间。在我国南 方, 虽然也有气候与环境都与该植物原产地类似的 地方, 但凤眼蓝大多漂浮生长于深水水体, 形成的种 子通常沉入深水，难以萌发。而生长于野外、有着水 位波动的浅水水体中的部分地方种群，可能存在着 一定水平的种子更新。海口一个种群内发现了实生 苗, 证明此种群既有种子形成，生境条件也适合种子 的萌发。

从地理分布来看, 发现有性繁殖的种群都分布 在华南和西南等地, 如发现了实生苗的海口以及种 群具有两种花型的重庆与南宁等地。这些地方与该 入侵植物原产地的气候以及生态条件(如传粉条件) 类似，且光照充分易满足种子萌发的条件，因此能够 实现一定水平的成功的有性繁殖 (形成自交或异交 种子并实现种子萌发)。由于有性繁殖使种群得以 通过种子进行扩散和长期休眠，促进了该植物的入 侵，所以，对这些地方入侵种群的管理控制应注意控 制有性繁殖。

\section{2 三型花柱的花型频率}

中国境内凤眼蓝的三型花柱系统都转化为 $\mathrm{M}$ 花型占据绝对优势, 许多种群甚至只有 $\mathrm{M}$ 花型 (图 2)。仅南宁和重庆的部分种群中出现了频率极低的 $\mathrm{L}$ 花型。中国境内种群没有发现 $S$ 花型 $(S$ 花型只出 现在凤眼蓝的原产地, 在全球所有入侵种群中都没 有被发现 (Barrett, 1989))。这种 M 优势”与 Barrett (1989)对全球凤眼蓝入侵种群的研究结果相一致。

是什么原因造成该入侵植物在世界范围的“ M 优势”呢? 这是一个引人注目却还悬而未决的问题。 $\operatorname{Barrett}(1977 ; 1989)$ 认为, 主要原因是 M 花型较其它 两花型有着较高的自交亲和性。但是, 凤眼蓝有性 繁殖水平很低, $\mathrm{M}$ 花型稍高的自交亲和性似乎难以 造成全球入侵种群如此显著的 M 优势。通过初步 的野外自然种群实验，本文作者提出了 $\mathrm{M}$ 优势可能 是由于 $\mathrm{M}$ 花型有着较高的克隆生长速率和潜力, 或 较强的耐寒性造成的”的假说，但目前还缺乏严谨的 实验证明，有关实验工作正在继续开展。

根据三型花柱的遗传基础 ( 2 个位点 $S 、 M$, 每个 
位点有两个等位基因。 $\mathrm{S}$ 控制的是 $\mathrm{S}$ 花型, M 控制 的是 M 花型。 S 位于 M 的上位 (Barrett ,1980b ) ) , L 花型的基因型为 ssmm, 很可能是通过在 $M$ 位点杂合 的 $\mathrm{M}$ 花型个体发生有性繁殖(主要是花内自交或同 株异花自交 (Geitonogamy) ) 后分离而产生的。当然, 重庆和南宁种群内出现的 L 花型是通过 $M$ 花型分 离而来, 还是由于再次引种带来的, 尚待进一步的研 究确定。

从地理分布来看, $\mathrm{L}$ 花型仅分布在两个地点: 重 庆和南宁, 这两地点都位于中国的西南地区。虽然, 目前还无法确定 L 花型是如何出现的, 但是经历了 多次引种的更大范围的其它地方没有发现有 L 花 型,所以, 这种 $\mathrm{L}$ 花型更可能是由于 $\mathrm{M}$ 有性繁殖分 离而来的。重庆和南宁等地具有有利于凤眼蓝有性 繁殖的气候和生态条件, 这也从一个侧面证实了这 一点。

\section{3 半同长花柱}

在中国 ,半同长花柱只出现在福州、南宁、海口 和重庆的种群内, 与有性繁殖水平和花型频率的地 理分布格局非常吻合。半同长花柱中, 柱头和花药 紧密靠近, 有着促进花内自交的潜力, 是对入侵生境 中花型不全、缺乏合适传粉者等导致异交难以进行 情况下的一种提高种子产量、实现繁殖保障的适应 (Barrett ,1979)。这种通过提高自交水平来适应入侵 生境的进化趋势与著名的 Baker 定律一致 (Baker， $1965 ; 1974)$,大大促进了该植物的入侵。不过, 由于 凤眼蓝种群有性繁殖水平非常有限, 半同长花柱的 变异个体难以在种群内扩散, 只能维持一个极低的
发生频率。

半同长花柱的地理分布格局与有性繁殖水平和 花型频率的地理分布规律非常吻合。半同长花柱是 由基因决定的，只能通过有性繁殖表达和强化，也反 过来促进了自交,所以有着半同长花柱变异的种群 往往有着较高的有性繁殖水平，同时也会通过有性 繁殖分离出 $\mathrm{L}$ 花型。这些繁育系统的变异集中出现 在少数几个地点的原因很可能是, 这些地区有着与 原产地类似的生境条件和较长的入侵历史, 使得这 几个地点种群易于实现有性繁殖和积累一定的遗传 变异。东北的盘锦和锦州, 北部的北京和淮安等地 的种群都只有 $\mathrm{M}$ 花型, 也没有发现花部特征上的显 著变异和有性繁殖迹象, 这是与其较近的入侵历史 (Ding et al，2000)和气候环境特点相联系的。

半同长花柱在雨久花科的多个植物中被发现， 在与凤眼蓝同属的 $E$. paniculata 中被研究得最为 深入 (Fenster \& Barrett ,1994)。E. paniculata 中的半 同长花柱是通过短雄惢群中的一个雄蕊伸长花丝而 实现的，受多个修饰基因 (Modifier genes)控制。这些 基因只在 $\mathrm{M}$ 花型内表达，且对花部其它特征没有明 显的表型影响(Fenster \& Barrett ,1994)。而在凤眼蓝 中 , $\mathrm{M}$ 花中的半同长花柱主要是由长雄荵群中的一 个雄芯缩短花丝造成的;也有可能是由花柱缩短造 成柱头与矮雄芯群靠拢 (福州种群)。凤眼蓝的半同 长花柱还可能通过 $\mathrm{L}$ 花型中的花柱缩短实现 (南宁 种群)。可见, 凤眼蓝中的半同长花柱变异可能受到 更多修饰基因控制，具有较 E. paniculata 更复杂的 遗传基础。

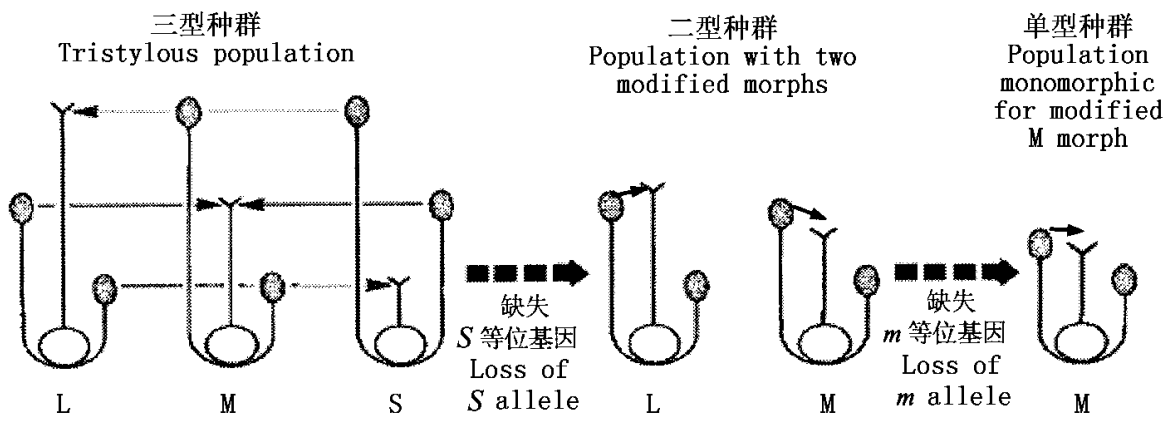

图 4 凤眼蓝中三型花柱向半同长花柱的进化

Fig.4 The evolution of semi-homostyly from tristyly in Eichhornia crassipes

箭头指示种群内主要的交配格局。注意二型种群内 $\mathrm{M}$ 花型长雄莈的变化和 $\mathrm{L}$ 花型中花柱的变化 The main mating processes are indicated by arrows. Note the modifications of long stamen in M or modification of styly in L floral morph 


\section{4 结 论}

与其它水生植物和入侵植物一样, 凤眼蓝以克 隆繁殖为种群主要更新方式。在有性繁殖方面，三 型花柱繁育系统作为一种异交传粉机制已经由于奠 基者效应造成花型缺失和入侵地的 不合适”传粉者 而中断; 高度的自交亲和性使得凤眼蓝的花内自交 也能结实。因此, 花部结构发生进化修饰产生半同 长花柱的变异个体能提高种子产量, 有着选择优势。 在这种情况下, 自交将是有性繁殖的主要方式。但 是种子萌发和幼苗建成的生态条件难以满足又进 一步限制了入侵种群的种子更新。由于有性繁殖水 平很低, 半同长花柱难以在种群内扩散, 只在少数存 在有性繁殖的种群内维持一个很低的频率。

从地理变化格局来看, 我国南方尤其是西南和 海南岛种群的繁育系统有着明显变化, 比其它地区, 尤其是北方地区种群的变异性要大得多。北方地区 种群的繁育系统单一, 主要以克隆生长为种群更新 方式; 而南方种群, 尤以西南和海南岛种群的繁育系 统最为多样化。究其原因, 很可能是南方部分地方 的生境条件并不适合凤眼蓝的生长, 尤其是冬季气 温较低, 凤眼蓝难以正常越冬 (凤眼蓝不耐寒冷, 通 常在水温 $7{ }^{\circ} \mathrm{C}$ 以上才能安全越冬, 日最低温度平均 值不低于 $-3{ }^{\circ} \mathrm{C}$ ) (周泽江等 ,1984)。海南岛和西南 部分地方充足的光照和较高的气温使得这些地方种 群有着一定的种子更新而维持了较多样化的繁育系 统。

雨久花科的其它三型花柱植物中也常常发现存 在着半同长花柱的变异花型 (Barrett ,1992)。除了前 面提到的 E. paniculata 以外, 与凤眼蓝同属的 $E$. azurea 中也发现了半同长花柱, 在 E. heterosperma， $E$. natans 和 $E$. diversifolia 中更是完全由半同长花 柱个体组成 (Barrett, 1992)。雨久花属 ( Pontederia) 中也发现了一个半同长花柱物种 (Pontederia parviflo$r a)$ 。这说明，凤眼蓝所在的雨久花科中三型花柱的 进化崩溃发生了多次, 每次都是向自交进化。对于 凤眼蓝这样的入侵植物, 繁育系统在入侵生境中的 这种适应性变化既是入侵造成的结果, 也在一定程 度上促进了入侵。

\section{参 考 文 献}

Baker, H. G. 1965. Characteristics and modes of origin of weed. In: Baker, H. G. \& G. I. Stebbins eds. The genetics of colonizing species. New York: Academic Press. $147 \sim 168$.

Baker, H. G. 1974. The evolution of weeds. Annuals Reviews of
Ecology and Systematics, 5: $1 \sim 24$.

Barrett, S. C. H. 1977. Tristyly in Eichhornia crassipes (Mart.) solms (Water Hyacinth). Biotropica, 9: $230 \sim 238$.

Barrett, S. C. H. 1979. The evolutionary breakdown of tristyly in Eichhornia crassipes (Mart.) Solms (Water Hyacinth). Evolution, 33: $499 \sim 510$.

Barrett, S. C. H. 1980a. Sexual reproduction in Eichhornia crassipes (Water Hyacinth) I. Fertility of clones from diverse regions. Journal of Applied Ecology, 17: $101 \sim 112$.

Barrett, S. C. H. 1980b. Sexual reproduction in Eichhornia crassipes (Water Hyacinth) II. Seed production in natural populations. Journal of Applied Ecology, 17: $113 \sim 124$.

Barrett, S. C. H. 1985. Ecological genetics of breakdown in tristyly. In: Haeck, J. \& J. W. Woldendorp eds. Structure and functioning of plant populations. Phenotypic and genotypic variation in plant populations. Amsterdam: North-Holland Publishing Company. $267 \sim 275$.

Barrett, S. C. H. 1989. Waterweed invasions. The Scientific American, 260: $90 \sim 97$.

Barrett, S. C. H. 1990. The evolution and function of heterostyly. Trends in Ecology and Evolution, 5: $144 \sim 148$.

Barrett, S. C. H. 1992. Genetics of weed invasions. In: Jain, S. K. \& L. W. Borsford eds. Applied population biology. Netherlands: Kluwer Academic Publishers. 91 119.

Barrett, S. C. H. 1993. The evolutionary biology of tristyly. In: Futuyma, D. \& J. Antonovics eds. Oxford surveys in evolutionary biology. Oxford: Oxford University Press. $283 \sim 326$.

Barrett, S. C. H. 2002. The evolution of plant sexual diversity. Nature Reviews Genetic, 3: $274 \sim 284$.

Darwin, C. 1877. The different forms of flowers on the plants of the same species. London: John Murray.

Ding, J. Q., R. Wang, W. D. Fu \& G. L. Zhang. 2000. Water hyacinth in China: its distribution, problems and control status. In: Julien, M. H., M. P. Hill, T. D. Center \& J. Q. Ding eds. Biological and integrated control of water hyacinth, Eichhornia crassipes. Proceedings of the second meeting of the global working group for the biological and integrated control of water hyacinth, Beijing, China, 9 12 October 2000. $29 \sim 32$.

Fenster, C. B. \& S. C. H. Barrett. 1994. Inheritance of matingsystem modifier genes in Eichhornia paniculata (Pontederiaceae). Heredity, 72: $433 \sim 445$.

Grace, J. B. 1993. The adaptive significance of clonal reproduction in angiosperms: an aquatic perspective. Aquatic botany, 44: $159 \sim 180$.

Guo, Y. H. (郭友好), S. H. Huang (黄双全) \& J. K. Chen (陈家宽). 1998. Breedingsystem and evolution of aquatic angiosperm. Acta Hydrobiologica Sinica (水生生物学报), 22: $79 \sim 85$. (in Chinese)

Heywood, V. H. (translated by Ke, Z. F. (柯植芬)). 1979.

Plant systematics. Beijing: Sciences Press.

Lee, C. E. 2002. Evolutionary genetics of invasive species. Trends 
in Ecology and Evolution, 17: $386 \sim 390$.

Li, B. (李博), B. S. Xu (徐炳声) \& J. K. Chen (陈家宽). 2001. Perspectives on general trends of plant invasions with special reference to alien weed flora of Shanghai. Biodiversity Science (生物多样性), 9: 446 457. (in Chinese with English abstract)

Li, Z. Y. (李振宇) \& Y. Xie (解炎). 2002. Invasive alien species in China. Beijing: China Forestry Publishing House. 1 54. (in Chinese)

Mack, R. N., D. Simberloff, W. M. Lonsdale, H. Evans, M. Clout \& F. Bazzaz. 2000. Biotic invasions: causes, epidemiology, global consequences and control. Issues in Ecology, $1 \sim 20$. Ren, M. X. (任明迅) \& D. Y. Zhang (张大勇). 2004. Herkogamy. In: Zhang, D. Y. ed. Plant life history evolution and reproduction ecology. Beijing: Science Press. $303 \sim 322$. (in Chinese)

Sax, D. F. \& J. H. Brown. 2000. The paradox of invasion.

Global Ecology and Biogeography, 9: $363 \sim 371$.

Silvertown, J. \& D. Charlesworth. 2001. Introduction to plant pop- ulation biology, 4th ed. Oxford: Blackwell Science.

Tang, P. H. (唐佩华), J. Z. Sun (孙金洲) \& Y. M. Liu (刘 一鸣). 1987. Studies on sexual reproductin of Eichhornia crassipes 1 . Getting seeds by pollination and seed germination. Acta Agronomica Sinica (作物学报), 13: $53 \sim 57$.

Watson, M. A. \& C. S. Cook. 1982. The development of spatial pattern in clones of an aquatic plant, Eichhornia crassipes solms. American Journal of Botany, 69: $248 \sim 253$.

Xu, C. Y. (徐承远), W. J. Zhang (张文驹), B. R. Lu (卢宝 荣) \& J. K. Chen (陈家宽). 2001. Process in studies on mechanisms of biological invasion. Biodiversity Science (生物多 样性), 9: 430 438. (in Chinese with English abstract)

Zhou, Z. J. (周泽江) \& J. H. Yang (杨景辉). 1984. The role of water hyacinth in sewage ecological treatment system and the approachs to their utilization. I. The biological characteristics of water hyacinths and the effects of environmental factors on their growth. Chinese Journal of Ecology (生态学杂志), 5: 36 40. (in Chinese)

责任编委 : 马克平 责任编辑 :刘丽娟

\section{欢迎订阅 2005 年的 Journal of Integrative Plant Biology (中文刊名:《植物学报》)}

《植物学报》由中国科学院植物研究所和中国植物学会主办, 是植物学综合性学术期刊。多年来一直被 国内 20 多个数据库和文摘期刊收录, 是中国科技信息研究所中国科技论文统计与分析数据库和中国科学院 文献情报中心中国科学引文数据库的来源期刊。多次荣获全国、中国科学院及科协优秀科技期刊奖。被引 频次和影响因子在我国生物类期刊中名列前茅。目前在国外发行和与国外交换的国家和地区达 40 多个。 被国外 40 多个英文文摘期刊收录, 1998 年进入美国科学信息研究所( ISI)数据库 SciSearch, 是 Biosis(生命科 学文献数据库)收集数据最多的中国期刊之一。本刊自 2005 年第一期起 将由国际著名的 Blackwell Publishing 出版, 英文名称为 Journal of Integrative Plant Biology”。

主要刊登 植物生理生化、结构植物学、植物分子生物学、植物遗传学、植物生殖生物学、植物生态学、植 物化学与资源植物学、植物系统与进化、古植物学以及生物技术等领域的原始研究论文、综述和快讯。国内 统一刊号 CN 11-5067/Q ,国际标准刊号变更为 ISSN 1672-9072,CODEN 码 CHWHAY, 国际标准 A4 大 16 开, 每 月 10 日出版，每期 128 页，铜版纸印刷。国内发行，定价每期人民币 90 元，全年 1080 元，国内邮发代号 2500 ,邮局订购。国外订购请与 Blackwell Publishing 联系。

编辑部地址 北京香山南辛村 20 号,邮编 100093

电话 : (010)62836563,), (010)82592636

传真 : (010) 82592636

E-mail :(jiaoyali@ibcas.ac.cn) 